\title{
DIÁLOGOS DE SABERES EN TORNO A RESTOS HUMANOS SENSIBLES. UNA PROPUESTA MUSEOGRÁFICA, AUDIOVISUAL Y EDITORIAL
}

\author{
DIALOGUES OF KNOWLEDGE ABOUT SENSITIVE HUMAN REMAINS. A \\ MUSEOGRAPHIC, AUDIOVISUAL AND EDITORIAL PROPOSAL
}

\author{
Mariana Fabra ${ }^{1,2}$ y Mariela Eleonora Zabala ${ }^{1,2}$
}

\begin{abstract}
Desde hace 15 años, el Programa de Arqueología Pública -PAP- (de la Secretaria de Extensión Universitaria, Facultad de Filosofía y Humanidades, Universidad Nacional de Córdoba) realiza trabajos bioarqueológicos en la costa sur de la Laguna Mar Chiquita (Córdoba, Argentina). Los pedidos de intervención fueron realizados por los trabajadores de los museos o vecinos, originados debido a las fluctuaciones hidrológicas de la laguna y en su impacto en sitios arqueológicos caracterizados por el hallazgo de restos humanos. Además de las actividades de rescate, se realizaron trabajos de capacitación y asesoramiento en propuestas patrimoniales. El objetivo de este trabajo es socializar una experiencia de Educación Patrimonial y Museológica que estamos construyendo junto con la comunidad del Museo de Ciencias Naturales Aníbal Montes -MCNAM- (Miramar, Córdoba, Argentina), algunos miembros de otros museos de la zona, representantes de los Pueblos Indígenas de Córdoba y el PAP. Esta experiencia de construcción colectiva, en un marco de diálogo de saberes, quedará plasmada en tres productos museológicos, museográficos y educacionales: una muestra itinerante, un documental y una publicación virtual. Interesa compartir las metodologías aplicadas para la recuperación de los saberes en torno al patrimonio arqueológico local, en particular restos sensibles tales como los restos humanos, así como el diseño de los guiones de cada producto incorporando las voces de múltiples actores sociales.
\end{abstract}

Palabras claves: multivocalidad, museografía, museología, educación patrimonial, arqueología pública.

For 15 years, the Public Archeology Program -PAP- (SEU, FFyH, UNC) has been carrying out bioarchaeological work on the southern coast of the Mar Chiquita lagoon (Córdoba, Argentina). The requests for intervention were made by the workers of the museums and neighbors as a result of the hydrological fluctuations of the lagoon and their impact on archaeological sites characterized by the discovery of human remains. In addition to the rescue activities, training and advisory work regarding cultural heritage projects were conducted. The objective of the present article is to socialize a Heritage Education and Museology experience that we are building together with the community of the Museum of Natural Sciences Aníbal Montes -MCNAM- (Miramar, Córdoba, Argentina), some members of other museums in the area, representatives of Indigenous Peoples of Córdoba, and the PAP. This experience of collective construction, within a framework of dialogue of knowledge, will be reflected in three museological, museographic and educational products: an itinerant exhibition, a documentary, and an online publication. We are interested in sharing the methodologies applied for the recovery of knowledge of the local archaeological heritage, particularly bioarchaeological heritage, as well as the design of the scripts of each product incorporating the voices of multiple social actors.

Key words: Multivocality, museography, museology, heritage education, public archaeology.

La Bioarqueología se ha consolidado en las últimas décadas como una disciplina que aporta conocimientos sobre los modos de vida de las poblaciones humanas a lo largo del tiempo a partir del estudio de restos óseos y dentales. Particularmente, permite entender, entre otras cuestiones, cuáles han sido los principales cambios y continuidades en la alimentación, el impacto que han tenido sobre la salud y la dieta procesos tales como el sedentarismo, la adopción de cultígenos, el contacto interétnico y la colonización europea, que de otro modo no se podrían conocer.

Pero, ¿cómo poner en diálogo los conocimientos que se generan sobre poblaciones humanas arqueológicas con los intereses de las propias comunidades -locales, indígenas- de donde proceden

\footnotetext{
${ }^{1}$ Instituto de Antropología de Córdoba, Museo de Antropología, Facultad de Filosofía y Humanidades, Universidad Nacional de Córdoba, Argentina. marianafabra@gmail.com; marielaeleonora@gmail.com

${ }^{2}$ CONICET. Consejo Nacional de Investigaciones Científicas y Técnicas, Buenos Aires, Argentina.
} 
los restos que se investigan? Entendemos a la Arqueología Pública como una disciplina que permite articular la Arqueología, la Educación y de manera particular, en nuestro caso, la Bioarqueología, ya que trabajamos especialmente con un tipo de materiales sensibles ${ }^{1}$ como son los restos óseos humanos. Siguiendo a Merriman (2004), la Arqueología Pública se presenta como una disciplina construida social y colectivamente por parte de distintos actores, crítica y reflexiva que tiene lugar en múltiples contextos. También, como una disciplina que estudia las relaciones entre la arqueología y la sociedad en el presente (Salerno 2013), integrando la reflexión sobre la propia práctica Arqueológica, la investigación y la participación social (Montenegro 2012).

En Argentina, el desarrollo sostenido y en aumento de líneas de investigación enmarcadas en esta disciplina desde una perspectiva biocultural, es un reflejo del interés creciente sobre este campo de estudio. Sin embargo, entendemos que este crecimiento no se ha visto acompañado del desarrollo de estrategias de difusión/extensión universitaria/divulgación de los resultados obtenidos en ámbitos científicos, salvo algunas excepciones (Aichino et al. 2013; Fabra y Zabala 2010, 2015; García Laborde 2017; Nahuelquir et al. 2015; Zabala y Fabra 2012; Zabala et al. 2006; 2016). Esto se debe, en parte, al poco reconocimiento que le otorga el ámbito académico a este tipo de actividades de intervención y extensión, incluyendo líneas de financiamiento para proyectos de extensión. Asimismo, estas propuestas educativas se encuentran muy dispersas, con poca o nula publicidad teniendo un impacto muy acotado en la sociedad en general.

En los últimos años, el Programa de Arqueología Pública-en adelante,PAP-harealizadonumerosas investigaciones en la costa sur de la Laguna Mar Chiquita (Córdoba, Argentina) enmarcadas en una perspectiva que entiende al registro esqueletal humano desde una perspectiva biocultural (Fabra et al. 2014a; 2014b) y pública de la Arqueología (Fabra y Zabala 2015; Zabala y Fabra 2012). Estos estudios, originados en hallazgos fortuitos de restos humanos (Fabra et al. 2008; 2014b), han posibilitado indagar acerca de la antigüedad del poblamiento humano en la región, la variabilidad morfológica craneofacial (Fabra y Demarchi 2013), los cambios y continuidades observados en las estrategias de subsistencia y las patologías orales vinculadas a las mismas (Fabra y González 2015), y las actividades realizadas con el cuerpo, a partir de cambios entesiales y degenerativos (Salega y Fabra 2017). También se han hecho estudios moleculares en poblaciones de distintas regiones de Córdoba, incluyendo el noreste de la provincia, que han permitido confirmar la persistencia de linajes amerindios maternos en la población (García y Demarchi 2009).

Desde el PAP, centramos nuestra atención sobre los derechos culturales que posee la ciudadanía, y que tienen relación con el acceso al conocimiento que se genera a partir del estudio y puesta en valor de distintas materialidades consideradas "patrimonios", tanto por la legislación provincial y nacional vigente, como por la comunidad científica. El concepto de patrimonio es una creación académica para dar cuenta de bienes, sitios, espacios, territorios o prácticas culturales que un grupo humano selecciona por su valor de representación en el aspecto histórico, inspiración creativa y/o natural. Siguiendo al antropólogo catalán Prats, el contenido que se genera en esta triangulación superpuesta puede posibilitar la selección de algún elemento potencialmente patrimonializable. Es decir, una cualidad que hay que potenciar, agenciar y activar (Prats 1997). En este caso particular, por tratarse de restos humanos, intervienen, además del mencionado, otras valoraciones, significaciones y sentidos, otorgados por los pueblos indígenas en el presente. Así, se nos plantea el desafío ¿cómo democratizar los saberes que se generan sobre este tipo particular de materialidades a diversos destinatarios?, ¿cómo integrar distintas valoraciones, significados, sentimientos y sentidos sobre estas materialidades en propuestas educativas y de divulgación/extensión universitaria/difusión científica?, ¿cómo se pueden generar nuevos saberes y modos de gestión de estas materialidades, desde una perspectiva multivocal?, ¿qué puede aportar la Bioantropología en la gestión y la creación de normativas para la custodia, conservación y documentación de estas materialidades?

En el presente trabajo nos interesa socializar y reflexionar acerca de prácticas extensionistas relacionadas con el estudio de restos humanos sensibles. Estamos interesadas en debatir, a partir de una experiencia concreta, distintas estrategias orientadas a la socialización de saberes generados sobre este tipo particular de restos, cuya investigación, divulgación y gestión genera debates éticos entre los profesionales, y entre éstos y los pueblos indígenas. Por tal motivo hemos decido estructurar el escrito dando cuenta de la secuencia temporal de las acciones.

Finalmente, interesa tratar de superar la dicotomía entre la Bioarqueología o la Arqueología Pública como disciplinas disociadas, integrando la investigación, la extensión, la transferencia, la conservación preventiva, la documentación y la gestión de este tipo particular de restos sensibles como partes de un mismo proceso, transitado en conjunto con los investigadores, las comunidades locales, los pueblos indígenas y los entes gubernamentales. 


\section{El Inicio del Trabajo de Campo con Comunidades Científicas, Locales e Indígenas "Caminando a orillas de la Laguna..."}

\section{Carlos}

Mari! aparecieron restos óseos en el camping, me aviso José Luis recién, los encontraron cuando salieron con Hugo a hacer el safari fotográfico

Mari

¡Hola! Gracias por avisar! Te pido vayas a la comisaria, como la vez anterior, y des aviso, así podemos ir en el marco del convenio

\section{Carlos}

¡Dale! Te aviso cuando vuelva de ahí

\section{Anahí}

Ya están los oficios

¿Pueden mañana?;

Mari podés organizar con Policía Judicial...ahora cuando entre el oficio le saco una foto y la mando

\section{Sole}

Yo puedo ir, también Darío, Paloma, Romi y Luciano

Mari

Llamo yo a Pol Jud x lo de La Para

Aldy

Mari

Recién vuelvo a hablar, todo organizado para salir mañana

\section{Sole}

OK, cambio y fuera, nos vemos mañana ${ }^{2}$

Así comienza el trabajo de campo, con una llamada telefónica o un mensaje vía redes sociales. Cuando iniciamos nuestro trabajo en 2005, la relación que se estableció en un primer momento con quienes nos convocaban estaba mediada por roles pre-establecidos: antropólogas/os, trabajadores de museos, vecinos/ as. Hoy, el "estar ahî", en palabras de Malinowski, el trabajo compartido en el campo y en los museos, en pos de conservar colecciones, así como el asesoramiento en las exhibiciones museográficas y los talleres brindados, ha generado una relación basada principalmente en la confianza y el conocimiento del otro, que se ha consolidado más allá de los tiempos urgentes de un rescate arqueológico.

Como ya señalamos, el trabajo se inicia a partir del hallazgo fortuito por parte de pobladores locales de restos óseos, que dan aviso a los museos municipales de la zona, o a trabajadores y voluntarios de los museos. Por tratarse de restos humanos, desde 2009 su recuperación se realiza en el marco de un convenio firmado entre Policía Judicial, el Equipo Argentino de Antropología Forense y el Museo de Antropología
-Facultad de Filosofía y Humanidades, Universidad Nacional de Córdoba (en adelante, MA) que posibilitó la creación de un equipo interdisciplinario que concurre al lugar del hallazgo, y determina si se trata de restos humanos o no, y si corresponden a población actual, histórica o arqueológica. Los miembros del PAP forman parte de este equipo, y una vez que se procede la exhumación de los restos, en colaboración con las comunidades locales (Figura 1), los mismos son trasladados al Instituto de Medicina Forense del Poder Judicial, donde se les da ingreso, y si son restos que corresponden a poblaciones arqueológicas, son llevados al Museo de Antropología, para su posterior estudio, acondicionamiento, inventario y conservación.

Hasta la fecha, hemos recuperado un total de 28 individuos de 12 sitios arqueológicos. Realizamos 18 fechados radiocarbónicos que permitieron estimar un rango de antigüedad de los mismos entre 4058 \pm 59 y $487 \pm 45$ AP. Los estudios bioarqueológicos han incluido el inventario, la determinación del sexo, la estimación de la edad de muerte, la estimación de la estatura y la lateralidad, el relevamiento de patologías orales y óseas, que puedan remitir a estrés nutricional, funcional y metabólico, así como traumas y cambios degenerativos y entesiales. Si bien una vez concluidos los análisis, a los museos se les entrega un informe que da cuenta de la información que se ha podido recuperar del análisis de estos restos, siempre manifiestan su interés y deseo por conocer más sobre estos sitios; además, estos informes están escritos con una terminología técnica, que puede complicar su lectura o interpretación a un público no especializado. Así, una vez concluidos estos estudios, nos preguntamos: ¿Cómo socializar y democratizar estos nuevos saberes? Dado que en la región que circunda la Laguna Mar Chiquita hay 27 museos agrupados en la Asociación Civil de Amigos del Patrimonio Histórico de Ansenuza, Suquía y Xanaes $^{3}$ (APHA), consideramos que una muestra museológica itinerante, un cortometraje documental y una publicación didáctica serían las opciones que permitirían una amplia circulación. Estas tres piezas se vinculan y complementan para potenciarse en una propuesta pedagógica museística, financiada por el Ministerio de Ciencia y Técnica del Gobierno de la Provincia de Córdoba ${ }^{4}$. La misma aborda como temática principal los cambios y continuidades en las formas de vida de las poblaciones que vivieron en la costa sur de la Laguna Mar Chiquita desde hace 5000 años, a partir de las investigaciones sobre restos humanos recuperados desde una perspectiva bioarqueológica. También trabaja la problemática de la emergencia de los pueblos y comunidades indígenas en Córdoba en el presente. 

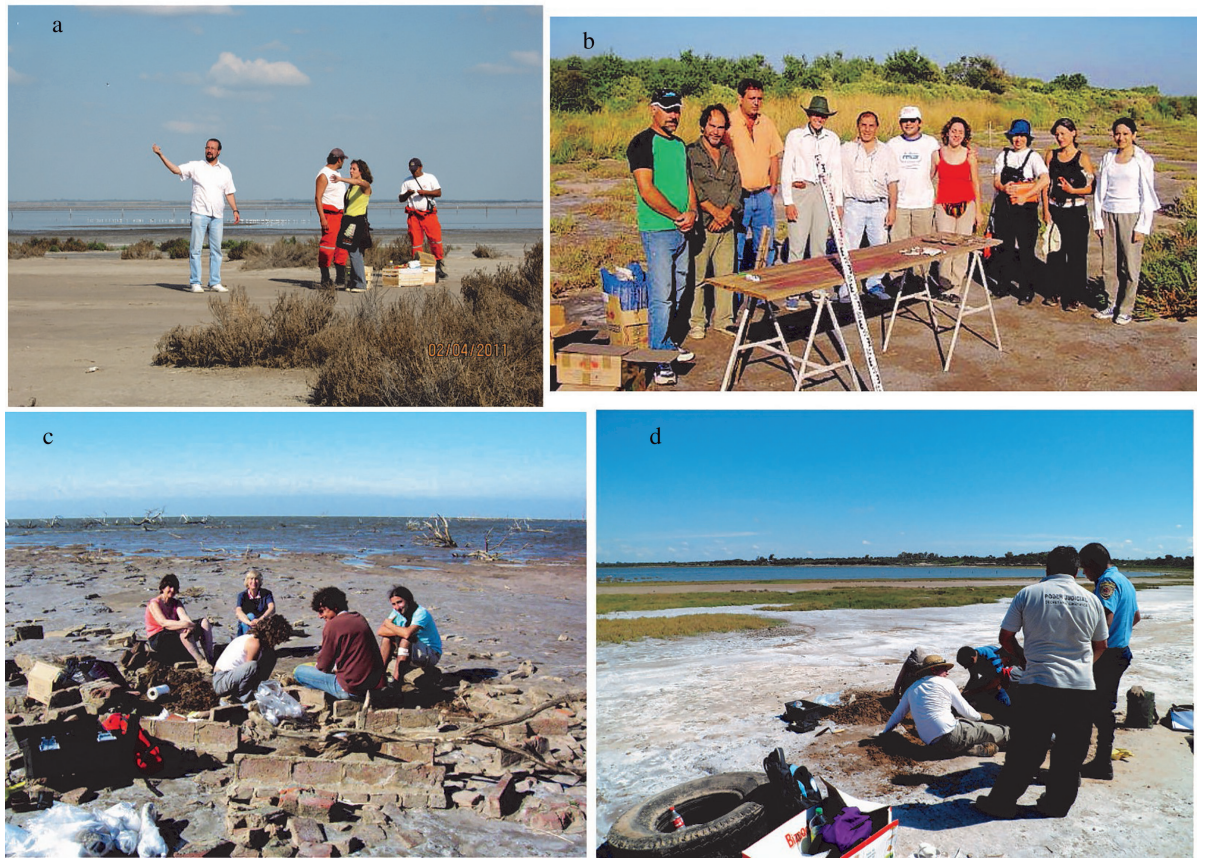

Figura 1. Algunos ejemplos del trabajo conjunto con miembros de comunidades locales: (a) sitio Isla Orihuela costa sudeste (2011), (b) sitio El Diquecito (2008), (c) sitio Colonia Müller (2006), (d) sitio 1387/15 La Para (2015).

Some examples of bioarchaeological work with members of local communities: (a) Isla Orihuela southeast coast site (2011), (b) El Diquecito site (2008), (c) Colonia Müller site (2006), (d) 1387/15 La Para site (2015).

Si bien este guión tiene como base los resultados de los trabajos bioarqueológicos realizados en la región, queremos destacar que su escritura incorporó los intereses y saberes que transmitieron los trabajadores de los museos de la región en dos talleres $^{5}$ (Figura 2a). La metodología elegida fue la de charla-taller (más adelante explicitamos la elección de la misma), mediante la cual los participantes escribían en afiches pegados a la pared o comentaban de manera oral sus conocimientos respecto a los modos de vida de los pueblos originarios de la región, tales como alimentación, vivienda, actividades cotidianas, creencias, tratamiento de sus difuntos, entre otros.

Asimismo, participó activamente el cacique Mario Frontera Tulian de la comunidad comechingona TacuKuntur (San Marcos Sierras, Córdoba, Argentina) (Figura 2b). Este guión será publicado en formato de $e$-book, de acceso libre, para que esté disponible a toda persona que quiera conocer en profundidad los contenidos desarrollados en la muestra. De esta manera, y desde una perspectiva pública de la Arqueología, entendemos que iniciamos un largo camino hacia la construcción multivocal de nuevos conocimientos y saberes en torno a los antiguos pobladores de la Laguna Mar Chiquita (Montenegro 2012).

El guión museográfico se compone de 10 carteles impresos sobre lona plástica (banderolas o banners)
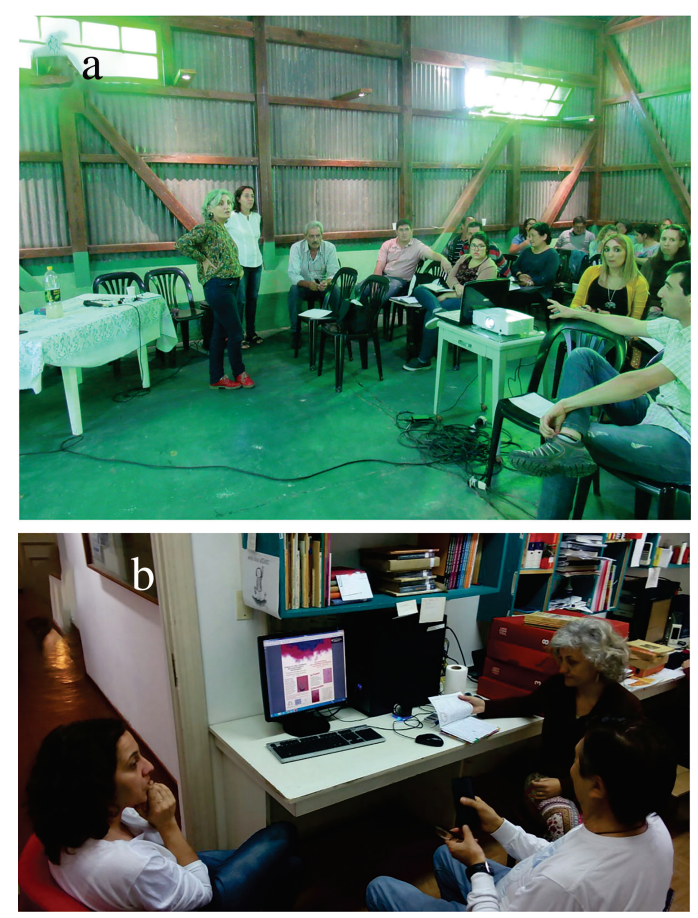

Figura 2. (a) Charla-taller realizada en la localidad de La Puerta, Córdoba. (b) Trabajo conjunto con Mario Frontera Tulián, comunidad comechingona Tacu Kuntur.

(a) Workshop held in La Puerta, Córdoba. (b) Working together with Mario Frontera Tulian, Tacu Kuntur, Comechingón community. 
autoportantes (sistema roll up), que permiten su fácil exposición en diferentes espacios públicos, en donde se presentan textos, mapas, escenas de la vida cotidiana ${ }^{6}$ y figuras humanas que sintetizan resultados bioarqueológicos para distintos momentos o períodos del Holoceno. También se relacionan las fluctuaciones hidro-climáticas de la laguna durante los últimos 5000 años con los cambios y continuidades en los modos de vida de las poblaciones. El cartel correspondiente al período más antiguo presenta información referida a las poblaciones que desarrollaron un modo de vida basado principalmente en la caza y la recolección (4500-2000 años AP); en otro cartel se discute la incorporación de prácticas de horticultura incipiente (2000-1000 años AP), para finalmente presentar cómo impactaron estos cambios en la dieta, la salud y usos del cuerpo de las poblaciones, sin reemplazar estrategias previas de caza y recolección (1000-300 años AP). Cada momento fue representado con un color particular, tomando como base la paleta de colores propia de los atardeceres en la Laguna Mar Chiquita ${ }^{7}$.

Pero ¿cómo reconstruir las historias de vidas de algunos de los individuos recuperados, sin que se pierdan dentro de una perspectiva más amplia, poblacional? Acá un recurso: las osteobiografías.

\section{Reconstruyendo Modos de Vida: Osteobiografías y Enfoques Poblacionales desde la Bioarqueología}

Desde fines de la década de 1970, la Bioarqueología ha generado valioso conocimiento acerca de poblaciones humanas a partir del análisis integral del esqueleto y la dentición humanas, vinculados a cada entorno y cultura particular (Martin et al. 2013). El interés principal estuvo focalizado en entender la adaptación humana desde una perspectiva biocultural (Larsen 2002), y abordar problemáticas vinculadas con la organización social, las estrategias de subsistencia, patologías vinculadas a estos cambios adaptativos, practicas mortuorias, entre otros.

Recientemente han surgido nuevos enfoques en esta disciplina, que sin abandonar la perspectiva integradora biocultural, centra su atención en el estudio de las ostebiografías humanas desde una mirada social que pretende entender las dimensiones culturales y sociales que construyen los cuerpos biológicos (Agarwal 2016). Estos enfoques postprocesuales proponen abordar temas usualmente no tratados en Bioarqueología, tales como el género, y otros aspectos de las identidades, individuales y colectivas.

De esta forma, se ponen en valor historias particulares, vidas singulares de personas, que de otro modo se desdibujarían en perspectivas poblacionales
(Joyce 2005). En nuestro país podemos mencionar el trabajo de Seldes (2012) y estudios osteobiográficos realizados por Scattolin y colaboradores (2005), Baffi y Seldes (2012) o Cortes (2010) como algunos antecedentes. En la provincia de Córdoba hemos incorporado recientemente perspectivas sociales en el estudio de restos humanos (Canova et al. 2017; Zarate et al. 2017), que se espera brinden información no sólo novedosa, sino complementaria, a la ya obtenida en estudios previos.

Volvamos a la exhibición. El guión museográfico se compone de diez carteles impresos sobre lona plástica autoportantes, de los cuales tres están destinados a presentar los estudios osteobiográficos realizados sobre seis individuos, dos por cada período, y de ambos sexos, con un doble objetivo: por un lado, presentar información que suele desdibujarse en torno a la vida de individuos singulares cuando la perspectiva es poblacional, y por otro, humanizar de alguna forma los relatos que construimos sobre las vidas de estas personas, basándonos en información científica, pero también en narrativas osteobiográficas ficticias (Boutin 2011:110). La selección de individuos de ambos sexos se justifica, según Mario Frontera Tulian (comunicación personal, 2016), en la cosmovisión comechingona de la dualidad de género, o Chacha-Warmi de origen andino, dualidad y complementariedad necesarias para equilibrar y armonizar la convivencia humana, o como dice Irigaray (1998), un puente entre naturaleza y cultura.

En los carteles donde se presentan las osteobiografías incluimos la información bioarqueológica, radiocarbónica, isotópica y genética, así como una recreación ficcional de sus actividades cotidianas. Estos textos se acompañan de dibujos en donde se reconstruye la figura humana, realzando aquellos aspectos que tienen que ver con posturas o actividades realizadas por esas personas en vida, así también como reconstruyendo de qué forma estas comunidades los inhumaron, al momento de su muerte $^{8}$ (Figura 3).

Recordemos que desde la Declaración de Arqueólogos en Río Cuarto (Córdoba) en el marco del Primer Foro de Pueblos Originarios (2005), se acordó no exhibir más restos óseos humanos en los museos, y este punto es ratificado enfáticamente por los representantes de las comunidades originarias de Córdoba ${ }^{9}$. Mario Frontera Tulian lo expresó de este modo en la Mesa de diálogo realizada en el MA en el marco del Día Internacional de los Museos, el 17 de mayo de 2017, bajo el lema "Decir lo indecible. Historias Controversiales”: “...en la ley de restitución hay dos cosas no negociables: no al tráfico y venta de restos; no a la exhibición y menos con fines de lucro. Estoy contento con el trabajo realizado. Hoy están en 

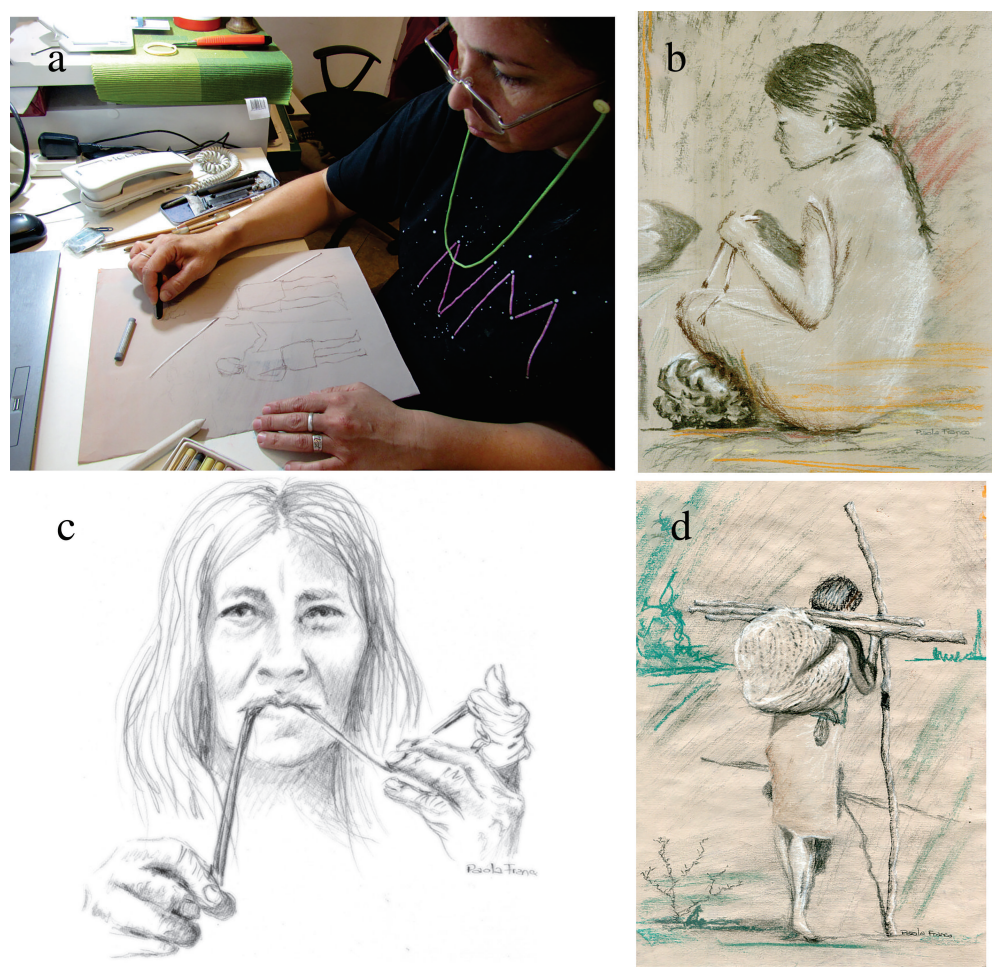

Figura 3. (a) Reconstrucción artística de osteobiografías realizada por Paola Franco. (b) "La Artesana" -mujer de entre 20 y 48 años de edad, 2562-47 años ${ }^{14} \mathrm{C}$ AP (AA93742)-, (c) "La Aldeana" -mujer de entre 43 y 58 años, 698-42 años ${ }^{14} \mathrm{C}$ AP (AA93743),- y (d) "La Viajera" -mujer de entre 45 y 49 años, 1911-59 años ${ }^{14} \mathrm{C}$ AP (MTC 14023)-.

(a) Artistic reconstruction of osteobiographies made by Paola Franco. (b) “The Artisan" -woman between 20 and 48 years old, $2562 \pm$ 47 years ${ }^{14} \mathrm{C} \mathrm{AP}\left(\mathrm{AA93742)}\right.$-, (c) "The Villager" -woman between 43 and 58 years old, 698-42 years ${ }^{14} \mathrm{C} \mathrm{AP}$. (AA93743),- and (d) "The Traveler" -woman between 45 and 49 years old, 1911-59 years ${ }^{14} \mathrm{C} \mathrm{AP}$ (MTC 14023)-.

el mejor lugar de conservación. Deben darse muchas condiciones para que se dé la restitución con seguridad y junto a ustedes".

La estrategia museográfica que encontramos para no exhibir restos humanos han sido las recreaciones artísticas. Nosotras acordamos con esta decisión tomada, y lo explicitamos en la cartelera poniendo las palabras de Mario Tulian. Esto viene en consonancia con el estudio de visitantes realizado en el Museo de La Plata en 2009 en el marco de la exhibición "Ser y pertenecer: un recorrido por la evolución humana". En esa ocasión los visitantes manifestaron que querían ver restos humanos pero acordaban con la decisión institucional de no exhibirlos (Reca et al. 2014).

La exhibición finaliza con un cartel que da cuenta de la presencia de los pueblos indígenas en la provincia de Córdoba ${ }^{10}$. Nos interesó abordar esta cuestión dado que en nuestra región de estudio las comunidades construyen su identidad a partir de la llegada del ferrocarril y la inmigración europea a fines del siglo XIX. En ese cartel presentamos las leyes nacionales y provinciales que reconocen la preexistencia de los pueblos indígenas a la creación del Estado Nacional. También mapeamos la ubicación de los pueblos indígenas reconocidos hasta el momento por el Instituto Nacional de Asuntos Indígenas (INAI) y el CPI en el territorio cordobés (Figura 4). Para ello contamos con el asesoramiento de Norma Sarasola, representante en el Consejo de Pueblos Indígenas en el INAI por la provincia de Córdoba, y con Horacio Pereyra, presidente del CPI de Córdoba. Por una cuestión de espacio físico en la cartelería fue una decisión museográfica sólo representar las comunidades reconocidas. Los procesos de comunalización de las mismas serán abordados en la publicación digital en formato de $e$-book, que contendrá el guión extendido de la muestra.

Hasta aquí hemos dado cuenta de las estrategias museográficas utilizadas para democratizar los conocimientos generados junto a algunos miembros de los pueblos originarios, pero faltaba dialogar con los trabajadores y vecinos de los museos de las localidades. Así fue como llevamos adelante charlastalleres con el objetivo de recuperar (a) conocimientos en torno a las materialidades que reconocían como parte de las comunidades originarias, (b) valoraciones y significaciones que dan a los restos humanos y (c) dar a conocer la propuesta museográfica, editorial y audiovisual. 


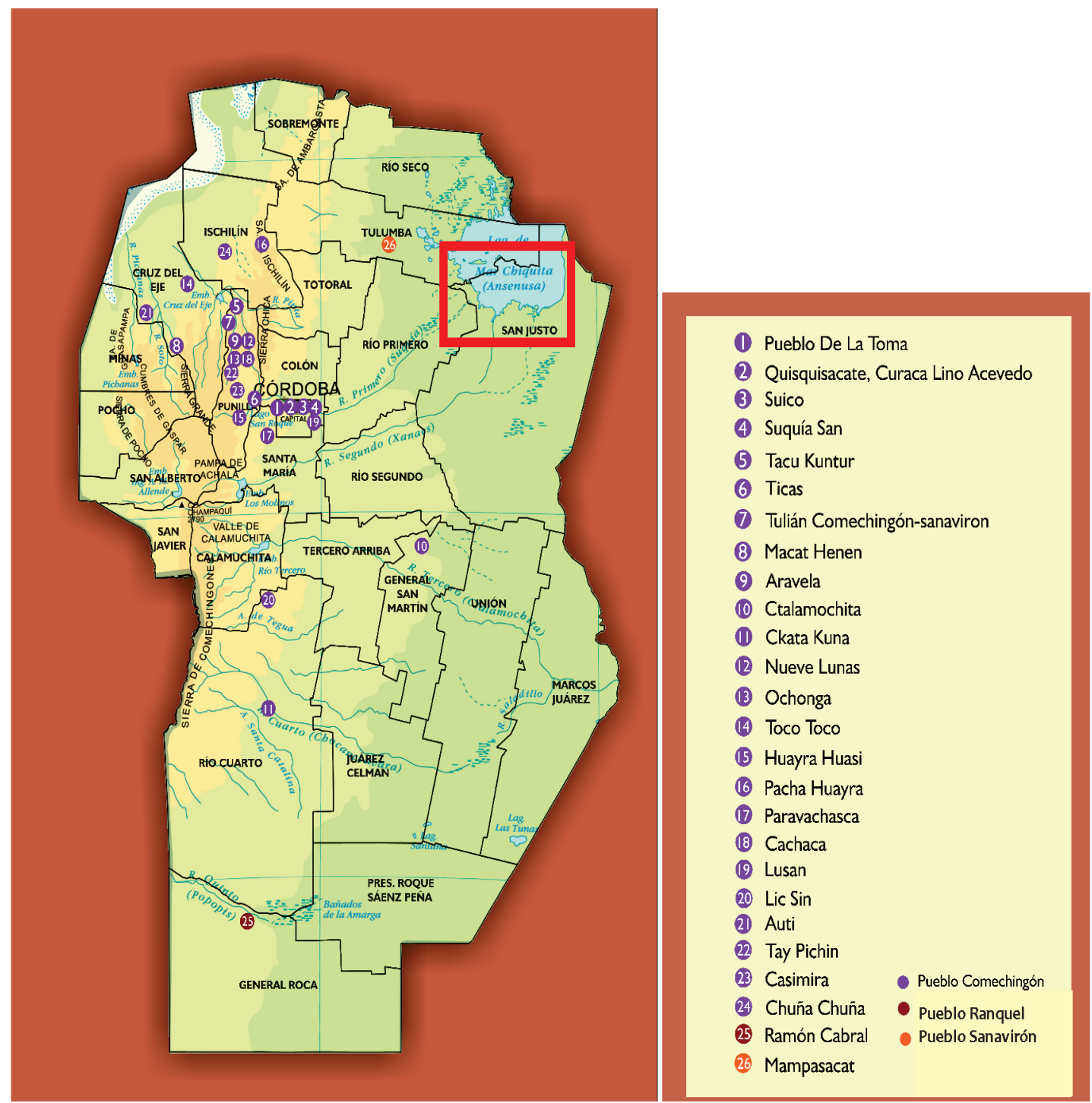

Figura 4. Mapa con comunidades de Pueblos Indígenas en la provincia de Córdoba (actualizado a diciembre de 2017). En recuadro rojo, la costa sur de la Laguna Mar Chiquita.

Map with communities of Indigenous Peoples in the province of Córdoba (updated to December 2017). In red box, the south coast of Laguna Mar Chiquita.

\section{Recuperando Saberes en Torno a los Restos Humanos \\ “QQué sabemos hoy de quienes vivieron aquí hace miles de años?"}

"El Museo de Miramar ha sido declarado de interés por el Ministerio de Educación de la Provincia de Córdoba y recomienda ser visitado en 4 grado del nivel inicial primario". (Juan José Scienza, Director del Museo, diciembre de 2016).
Así es como nos cuenta Juan José las motivaciones por las cuales el Museo recibe tanto público escolar en los meses de septiembre, octubre y noviembre. Durante esos meses, son visitados por contingentes de alumnos que se encuentran cursando el 4 grado del nivel primario, donde se trabaja como eje "Las sociedades y los espacios geográficos", y como delimitación espacial "La Provincia de Córdoba en el Contexto Nacional" (Diseño Curricular de la Provincia de Córdoba, 2012-2015:138). Entonces, no son solo niños de la localidad si no de toda la Provincia de Córdoba los que visitan el Museo. 
Al recorrer el Museo de Ciencias Naturales de la región de Ansenuza "Aníbal Montes" (en adelante, MCNAM) lo primero que sorprende es la inequidad en la exhibición de la presencia de los pueblos originarios con respecto al "ambiente natural". Es decir, se ha dedicado más espacio a las aves, al flamenco rosa y el ambiente que al modo de vida de los antiguos pobladores de la Laguna (D'Agostino 2015). Este es un museo de gestión asociativa de vecinos, -entre ellas muchas docentes- interesados en el patrimonio de la localidad, es decir no problematizan, ni conceptualizan a los individuos indígenas como "objetos de estudio". Entonces nos preguntamos ¿a qué se debe esta decisión museológica, cuando el PAP lleva más de 10 años trabajando en la zona a partir de los hallazgos fortuitos que ellos hacen? Ante esta inquietud surgió la necesidad como Programa de trabajar colaborativamente en la realización de la muestra itinerante, el video y la publicación educativa centrada en los pueblos originarios ayer y hoy. ¿Cómo hacerlo?

El modo que implementamos para la construcción conjunta del guión de la museográfico fue la de "charla-taller", dado que es una metodología de trabajo que posibilita el diálogo, la conversación y el intercambio de saberes para transformar y recrear nuevos conocimientos (Quezada et al. 2001). La clave organizativa es la problematización en acción, ya que permite un hacer creativo y reflexivo poniendo en juego marcos conceptuales desde los cuales se llevan a cabo las actividades y/o se van generando otros nuevos. Asimismo, permite integrar saberes en el trabajo colectivo y colaborativo, las vivencias, las reflexiones, el intercambio, la toma de decisiones y la elaboración de propuestas en equipos de trabajo.

Incorporamos la modalidad de charla para exponer nuestra propuesta: "Vida y muerte en el Mar de Ansenuza: un relato desde la Bioarqueología. Creación de una muestra itinerante". Cada charla-taller fue planificada en tres momentos. En el primero, se realizó la presentación de los participantes, y se comentó la modalidad de trabajo (Figura 5a, c). En el segundo, se colocaron afiches con las siguientes preguntas disparadoras: ¿Qué conocen de los modos de vida pasados en esta región?, ¿de dónde venían los primeros pobladores?, ¿cuándo llegaron?, ¿qué comían?, ¿cómo vestían?, ¿dónde vivían?, ¿qué hacían?, ¿qué idioma hablaban?, ¿cómo se llamaban? Estos afiches podían ser completados por los participantes del encuentro, de forma individual y/o grupal (Figura $5 b, d$ ).

Luego, se leyeron sus producciones, generamos preguntas acerca de qué más querían conocer sobre los pueblos indígenas y contamos la propuesta museográfica, audiovisual y didáctica. Finalmente, en el tercer momento presentamos algunos resultados de los trabajos Bioarqueológicos realizados en la zona. Todo el taller fue registrado a través de tomas de notas y fotografías.
En la charla-taller brindada en Miramar en diciembre de 2016 asistieron 17 participantes, entre ellos los propios trabajadores del Museo Aníbal Montes, guías y miembros de la comisión directiva actual, así como anteriores. También trabajadores del Museo Fotográfico de Miramary del Museo de Altos de Chipión, docentes del nivel primario de la Escuela Alberdi (Miramar) y de nivel inicial de la localidad de Balnearia, miembros de la Red de Institutos de Estudios Históricos de la Provincia de Córdoba (RIEC) y de la Asociación de Amigos del Patrimonio Histórico de Ansenuza (APHA).

Algunos de los temas que emergieron fueron acerca de los inmigrantes, así como también del interés por conocer la historia sobre los pueblos de la zona, inclusive sobre los afrodescendientes; $\sin$ embargo, este último tema no pudo ser abordado, ya que no forma parte de las líneas de investigación del PAP. Surgió como inquietud, a modo de reflexión, la posibilidad de incluir en la muestra las implicancias bioéticas y patrimoniales de trabajar con restos humanos, de valor patrimonial "para arqueólogos". De los afiches confeccionados, recuperamos ideas y conceptualizaciones que fueron incorporadas al guión museográfico, como, por ejemplo: Eran enterrados de manera ritual a la orilla de la laguna; ¿eran predadores o convivían conservando el medio ambiente? El diálogo se disparaba a través de estas preguntas, así como ampliando algunos puntos de su interés.

Un aspecto que nos parece importante destacar, es que los participantes no conocían la existencia de pueblos indígenas en el presente. De allí que decidimos incorporar a la muestra un cartel que hable sobre la situación actual de estos pueblos en Córdoba, a nivel nacional y provincial en su vínculo con la ley.

En la charla-taller realizada en la localidad de La Puerta en marzo de $2017^{11}$ asistieron 13 trabajadores de los museos de las localidades de La Puerta, Marull, Paso de las Tropas, San José de la Dormida, La Para, Las Peñas, Obispo Trejo y Las Arrias. Los temas que surgieron fueron la falta de conocimiento acerca de cómo proceder cuando aparecen restos humanos; la existencia en las escuelas de colecciones, ya que los niños cuando aparece algo allí lo llevan. Los saberes sobre los pueblos indígenas en pasado versaban sobre alimentación, la fauna existente y la alfarería. Pero a diferencia del taller anterior, a éste asistió un miembro del Pueblo Comechingón y pudo contar en primera persona sus procesos de re-emergencia étnica y reivindicación en el presente. Walter se presentó diciendo que "era del Museo de San José de la Dormida y estaba participando de la comunidad Comechingona de San Marcos Sierras porque en su zona no había pueblo originario organizado". Nuevamente se expuso la falta de comunidad originaria en la región y la carencia de conocimiento acerca de los mecanismos previstos por la ley para su autoreconocimiento. 


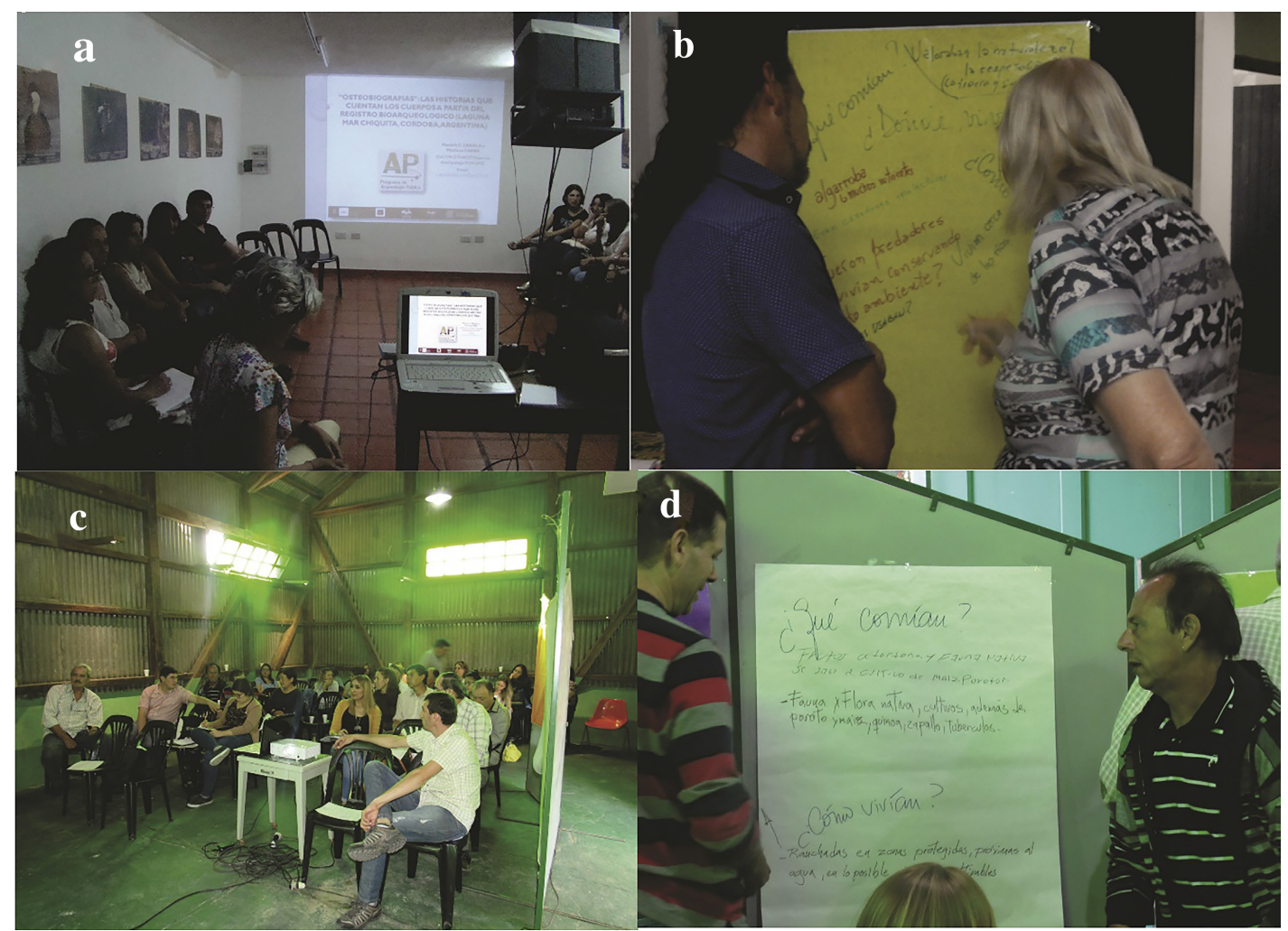

Figura 5. Charlas-talleres: (a, b) Museo de la región de Ansenuza, Aníbal Montes, diciembre de 2016, (c, d) Museo Histórico Municipal La Puerta, marzo de 2017.

Workshops: ( $a, b)$ Museo de Ciencias Naturales de la región de Ansenuza, Aníbal Montes, December 2016, (c, d) Museo Histórico Municipal de La Puerta, March 2017.

Acerca de los sitios excavados por el PAP, plantearon el deseo de conocer dónde estaban ubicados a fin de generar un sentido de pertenencia e identidad a través de una historia en común. En función de esta demanda se incluyó en la muestra mapas donde se grafican las fluctuaciones hidroclimáticas de la laguna a lo largo del tiempo, así como los sitios estudiados. De este modo fuimos recuperando saberes e interés de los participantes para hacerlos partes del guión museográfico. La creación de la agenda en común tuvo como fin gestionar la exhibición, es decir la circulación, el traslado, el costo y un cronograma de presentaciones.

\section{El Trabajo Arqueológico y Bioantropólogico Visto en Perspectiva Audiovisual y Multivocal}

La muestra está acompañada por un cortometraje documental, en el cual se aborda la metodología de trabajo que fuimos desarrollando como equipo en los últimos años, vinculando la universidad a través de su política extensionista, el Poder Judicial de Provincia de Córdoba y los museos locales. El cortometraje tiene como ejes (a) discutir la importancia del trabajo de las/ os arqueólogas/os y bioantropólogas/os en el rescate de restos óseos humanos hallados de manera fortuita, así como (b) debatir entre distintos actores sociales cómo deberían ser tratados, conservados y nombrados los restos humanos, y (c) cuál debería ser el destino final de los mismos.

Interesó destacar en el cortometraje la realización de estas prácticas de intervención cultural y social desde una universidad pública, así como reflexionar acerca de las significaciones y valoraciones otorgadas por distintas comunidades (arqueólogos, estudiantes, miembros de pueblos originarios, trabajadores de museos) al trabajo sobre restos humanos, y por sobre todo, dar cuenta del número de actores sociales e instituciones que interactúan cuando entendemos a la arqueología como una práctica pública.

Pero ¿cómo dar cuenta de todos estos temas y de la diversidad de actores sociales e instituciones involucradas en la práctica arqueológica? Si bien asesoramos en su contenido, la realización del guión, de la filmación y rodaje está a cargo de la cineasta Daniela Goldes ${ }^{12}$. Este cortometraje documental dura 45 minutos y es de libre acceso a través de los canales que posee la Facultad en un portal de internet ${ }^{13}$.

A nuestro cargo estuvo la selección y el contacto con los entrevistados a fin de dar cuenta del complejo 
entramado social del que somos parte al momento de rescatar restos humanos de carácter sensible. Ya no es suficiente contar con "el permiso para excavar" otorgado por la Agencia Córdoba Cultura del Gobierno de la Provincia de Córdoba, hoy el Consejo de Comunidades de Pueblos Indígenas de la Provincia de Córdoba “... entenderá en todas las cuestiones en la que se vean involucrados -directa o indirectamente- intereses de las comunidades..." (Ley 10316 art 4). Es decir, antes de intervenir, y decir qué hacer con los restos humanos, deben ser puestos en conocimiento.

Fue nuestra tarea diseñar el cuestionario, así como elegir los espacios de filmación para mostrar el entramado institucional que permite que hoy podamos recuperar restos humanos.

Este cortometraje documental podrá ser utilizado también en la formación de grado de los estudiantes de Antropología, porque exhibe de una manera muy didáctica un nuevo paradigma ético-científico del trabajo con restos humanos. De este modo, la extensión, la investigación y la formación de grado, pilares de la formación universitaria, se retroalimentan y enriquecen formando profesionales comprometidos con la sociedad de la que son parte.

\section{Una Propuesta Editorial De esto los libros nada dicen...}

Esta expresión fue manifestada en reiteradas oportunidades por los docentes que asisten a nuestros talleres, donde si bien agradecen la formación recibida, demandan bibliografía actualizada sobre los temas tratados. Desde el PAP hemos desarrollado una línea editorial que cuenta con tres números, con el fin de llegar al público escolar con nuevos conocimientos sobre educación patrimonial, pedagogía museística (Zabala y Roura Galtes 2007; Zabala et al. 2006) y los pueblos originarios de Córdoba (Fabra y Zabala 2010). Estos se encuentran en formato papel y digital de acceso libre en el Repositorio Digital Ansenuza ${ }^{14}$.

El guión museológico de esta exhibición será publicado en formato de libro electrónico, de difusión libre y gratuita, con el objetivo de posibilitar a los interesados mayor información sobre las investigaciones que sustentan la muestra. También se publicará un cuaderno didáctico para docentes con propuestas de secuencias didácticas para el aula. Siguiendo a Pastor Homs (2004) queremos abordar la visita a la exhibición como una práctica pedagógica museística que contemple tres momentos: la pre visita, la visita y la post visita. Es decir, que el docente trabaje los contenidos de la muestra que visitará con su grupo-clase en el aula, en el museo y nuevamente en el aula. De esta manera, el docente deberá conocer el museo y la exhibición antes de llevar a sus estudiantes para poder construir su secuencia didáctica buscando que la visita sea más significativa en el proceso de enseñanza y aprendizaje.
Interesados en hacer una muestra inclusiva, teniendo en cuenta la diversidad cultural y las minorías lingüísticas y sordas, el documental será doblado en lengua de señas y los textos de la exhibición así como la publicación con el guión museológico y el cuaderno didáctico serán subidos a la plataforma TIFLOlibros.

\section{A Modo de Conclusión: Comienza la Itinerancia}

La muestra museográfica y el cortometraje audiovisual fueron inaugurados el día 23 de marzo de 2018, en el MCNAM. Hasta el momento, ha circulado por siete museos ${ }^{15}$ del Noreste provincial, y tiene previsto continuar su itinerancia hasta mediados de 2019 (Figura 6).

A lo largo del artículo hemos querido reconstruir una propuesta extensionista, comunicacional, museográfica y museológica materializada en una muestra itinerante, un cortometraje documental y una propuesta editorial, estos dos últimos de acceso abierto en línea. El guión museológico es fruto de investigaciones bioarqueológicas así como de conversatorios en distintos formatos, entrevistas individuales y talleres con distintos actores sociales. En nuestras investigaciones, consideramos a los restos humanos no como objetos o materialidades de exclusivo interés arqueológico, sino como personas (Guichón 2016) o restos sensibles, en el sentido que le dan los pueblos indígenas a los restos de sus ancestros.

La creación de estos productos nos permitió revincularnos con la comunidad científica, local, indígena y artística en una propuesta de educación museística novedosa. En estos múltiples y diversos encuentros desarrollamos la "reflexividad", como docentesinvestigadoras de una universidad pública, donde la Antropología como oferta académica tiene solo 17 años. Nuestra reflexividad fue facilitada por el propio marco teórico y la metodología con el cual abordamos la investigación y la construcción de la exhibición, el documental y la publicación, como es la Arqueología Pública (Montenegro 2012). Además, el diálogo que se produjo entre las distintas comunidades interesadas en el estudio de los restos humanos permitió construir nuevos saberes que no se sustentaron en el conflicto, como ocurrió en otros casos (Curtoni y Paredes Mosquera 2014), sino en el reconocimiento de la diversidad de miradas y la potencialidad de las mismos para visibilizar a los pueblos indígenas, ayer y hoy.

En cada encuentro con ese "otro" nos presentamos como docentes e investigadoras, y a la Arqueología Pública y la Bioarqueología, como disciplinas que nos permiten reconstruir modos de vida a partir del estudio de restos humanos. Discutimos sus alcances y limitaciones. Ningún encuentro fue igual porque cada comunidad disponía de tiempos distintos, y participaban actores con distintos intereses. Porque tuvimos que aprender que leer y escribir es una competencia muy nuestra, pero no así de 


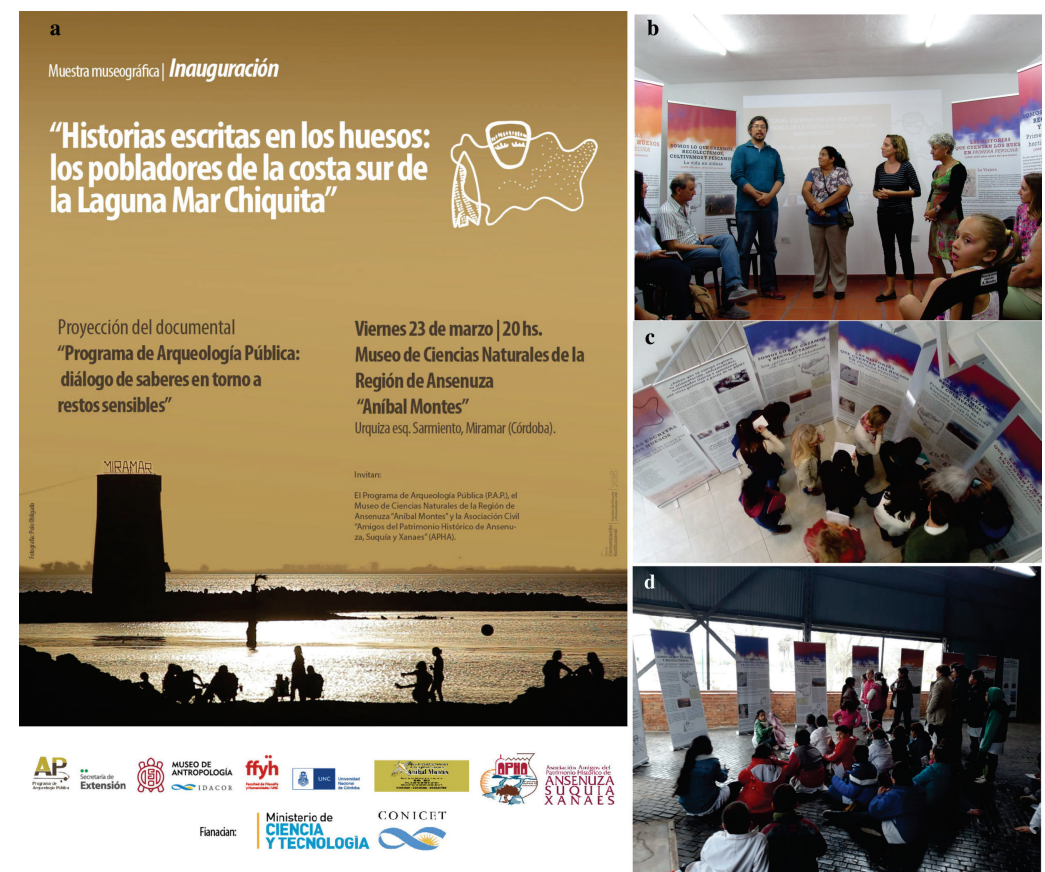

Figura 6. (a) Flyer de inauguración de la muestra en MCNAM, (b) palabras brindadas por miembros de comunidades indígenas de Córdoba durante la inauguración de la muestra, de izquierda a derecha: Cristian Bustos, de la comunidad Mampa Sacat (Pueblo Sanavirón) y Julia Ceballos, de la comunidad Aravela (Pueblo Comechingón), Mariana Fabra y Mariela Zabala, (c) inauguración en Museo Histórico Municipal de Villa del Rosario (agosto 2018), (d) visita guiada a la muestra por parte de alumnos de nivel primario, en el Salón de Usos Múltiples de la localidad de La Para (julio 2018).

(a) Exhibition opening flyer in MCNAM, (b) words given by members of the indigenous communities of Cordoba during the opening of the exhibition, from left to right: Cristian Bustos, from the Mampa Sacat community (Pueblo Sanavirón) and Julia Ceballos, from the Aravela community (Pueblo Comechingón), Mariana Fabra and Mariela Zabala, (c) inauguration in the Municipal Historical Museum of Villa del Rosario (August 2018), (d) guided tour of the exhibition by primary school students, in the multipurpose room, La Para town (July 2018).

nuestros interlocutores. Porque no todos los participantes manejaban las mismas herramientas comunicacionales. Y lo más importante, porque había desconfianzas que debíamos transformar en confianzas, para poder trabajar.

Entendemos a esta construcción multivocal de saberes en el sentido planteado por Curtoni y Paredes Mosquera (2014), alejada de las neutralidades, y asumiendo la posibilidad de disensos, cuestionamientos y diálogos en donde no todos los actores están en igualdad de condiciones. Donde se valoricen los distintos tiempos necesarios para construir esos saberes, local y temporalmente situados, la importancia de los vínculos entre los actores (Guichón 2016) y la construcción de espacios de confianza (Guichón et al. 2015). Y también, siguiendo a Menezes Ferreyra et al. (2014) una construcción multivocal en donde las comunidades involucradas sean participes también de las tomas de decisiones en torno a la gestión de sus patrimonios y la difusión de narrativas sobre el pasado.

Agradecimientos: A los voluntarios, trabajadores y amigos de los museos públicos de la región de Ansenuza por su compromiso y participación en las actividades propuestas. A quienes acompañaron el trabajo de producción de la propuesta museográfica, audiovisual y editorial. Muy especialmente a Horacio Pereyra (comunidad comechingona Toco Toco) y Mario Frontera Tulián (comunidad comechingona Tacu Kuntur), por su interés y apoyo constante. A Norma Sarasola (comunidad comechingona Ctalamochita) por su asesoramiento sobre las comunidades indígenas, a Mariana Minervini por la confección del mapa y a Paola Franco por las figuras que ilustran las osteobiografías. A Cristian Bustos, de la comunidad Mampa Sacat (Pueblo Sanavirón), Carlos Loza y Julia Ceballos, de la comunidad Aravela (Pueblo Comechingon) por acompañarnos en la inauguración. A Carlos Ferreyra, y por su intermedio, a la Asociación Civil de Amigos del Patrimonio Histórico de Ansenuza, Suquía y Xanaes (APHA) por las gestiones realizadas que facilitaron el traslado de la muestra a lo largo de 2018. A la Secretaría de Extensión Universitaria (FFyH, UNC), especialmente a Carlos Szulkin. A nuestras colegas Mónica Montenegro, Alejandra Pupio y María Eugenia Conforti por su ayuda en la búsqueda de antecedentes. A Paloma Zárate, Julieta Bellis y Valeria Martín por su colaboración en el registro realizado en los talleres. A los evaluadores anónimos y editores de la revista, por sus valiosos comentarios. 


\section{Referencias Citadas}

Agarwal, S. 2016. Bone morphologies and histories: Life course approaches in bioarchaeology. Yearbook of Physical Anthropology 159:130-149.

Aichino, G.L., C. De Carli, M. Zabala y M. Fabra 2013. Mapeando el patrimonio arqueológico de Córdoba. 2013. Propuesta educativa para el nivel medio con orientación en ciencias sociales y humanidades. Ansenuza, Repositorio de materiales educativos para la formación y el desempeño docente, https://ansenuza.unc. edu.ar/comunidades/handle/11086.1/761 (27 de diciembre de 2018)

Baffi, I. y V. Seldes 2012. La mujer en el registro bioarqueológico y su visibilidad en los contextos funerarios. Comechingonia Virtual: Revista Electrónica de Arqueología 16 (1):53-70.

Boutin, A.T. 2011. Crafting a bioarchaeology of personhood Osteobiographical narratives from Alalakh. En Breathing New Life into the Evidence of Death: Contemporary Approaches to Bioarchaeology, editado por A. Baadsgaard, A.T. Boutin y J.E. Buikstra, pp. 109-133. School for Advanced Research Press, ciudad.

Canova, R., S. Salega, C. González y M. Fabra 2017. Reconstrucción de las historias de vida de dos individuos (sitio El Diquecito, Laguna Mar Chiquita, Córdoba): una aproximación osteobiográfica. Libro de Resúmenes XIII Jornadas Nacionales de Antropología Biológica, compilado por R.A. Guichón, J.M. Motti, P. García Laborde, M. Martucci y L.O. Valenzuela, pp. 189, Asociación Argentina de Antropología Biológica, Necochea.

Cortes, L.I. 2010. Cuerpos en contraste: reflexiones sobre el tratamiento de los difuntos en dos entierros de 3.000 años A.P (Valle del Cajón, Noroeste argentino). Revista del Museo de Antropología 3:5-12.

Curtoni, R. y A. Paredes Mosquera 2014. Arqueología y multivocalidad en la encrucijada aportes críticos desde Sudamérica. En Multivocalidad y Activaciones Patrimoniales en Arqueología: Perspectivas desde Sudamérica, editado por M.C. Rivolta, M. Montenegro, L. Menezes Ferreira y J. Nastri, pp. 89-109. Fundación de Historia Natural Félix de Azara, Buenos Aires.

D’Agostino, L. 2015. Representaciones y significaciones de la Arqueología y Antropología en los visitantes y trabajadores del Museo de Ciencias Naturales de la región de Ansenuza, "Aníbal Montes" (Miramar, Córdoba). Proyecto de tesis de licenciatura, Universidad Nacional de Córdoba, Córdova.

Declaración de Rio Cuarto. 2005. Arqueología Suramericana 1 (2):287-288.

Diseño Curricular de la Provincia de Córdoba, 2012-2015:138 http://www.igualdadycalidadcba.gov.ar/SIPEC-CBA/ publicaciones/EducacionPrimaria/DCJ_PRIMARIO\%20 23\%20de\%20noviembre.pdf (27 de diciembre de 2018).

Endere, M.L. y P. Ayala 2012. Normativa legal, recaudos éticos y práctica arqueológica. Un estudio comparativo de Argentina y Chile. Chungara Revista de Antropología Chilena 44 (1):39-57.

Fabra, M. y D.A. Demarchi 2013. Análisis morfogeométrico aplicado al estudio de los patrones espaciales y temporales de variación morfológica craneofacial en poblaciones del centro de Argentina. Revista Cuadernos del Instituto Nacional de Pensamiento Latinoamericano 1 (1):87-101.
Fabra, M. y C.V. González 2015. Diet and oral health of populations that inhabited central Argentina (Córdoba province) during late Holocene. International Journal of Osteoarchaeology 25:160-175.

Fabra, M., R. Nores, S. Salega y C.V. González 2014a. Entre las sierras y el Mar: investigaciones bioarqueológicas en el noroeste de la región pampeana (costa sur Laguna Mar Chiquita, Córdoba, Argentina), En Avances recientes en la Bioarqueología Sudamericana, editado por L. Luna, C. Aranda y J. Suby, pp. 205-231. Grupo de Investigación en Bioarqueología, Buenos Aires.

Fabra, M., S. Salega, C. González, R. Smeding y E. Pautassi 2008. Arqueología de rescate en la costa sur de la Laguna Mar Chiquita: sitio arqueológico El Diquecito. Memorias del Pueblo: Revista del Museo Histórico Municipal La Para 8 (8):37-46.

Fabra, M., S. Salega, C.V. González y A. Tavarone 2014b. Lo que el agua nos dejó: investigaciones bioarqueológicas en la costa sur de la laguna Mar Chiquita (Córdoba, Argentina). Jangwa Pana 13:51-64.

Fabra, M. y M. Zabala 2010. Pueblos de las Sierras, del Piedemonte, de los Valles y la Llanura: la Protección del Patrimonio Arqueológico de las Sociedades Indígenas de Córdoba. Museo de Antropología, Universidad Nacional de Córdoba, Córdova.

Fabra, M. y M. Zabala 2015. Humanidad, Patrimonio, Ancestros: ¿de qué hablamos cuando hablamos de Arqueología Pública en Córdoba? En La Arqueología Pública en Argentina: Historias, Tendencias y Desafíos en la Construcción de un Campo Disciplinar, editado por M. Fabra, M. Montenegro y M. Zabala, pp. 53-75, Editorial de la Universidad Nacional de Jujuy, Jujuy.

García, A. y D.A. Demarchi 2009. Incidence and distribution of Native American mtDNA Haplogroups in Central Argentina. Human Biology 81 (1):59-69.

Garcia Laborde, P. 2017. Estado Nutricional de la Población Selk'nam: Aproximación Bioarqueológica al Impacto Generado por la Misionalización. Misión Salesiana Nuestra Señora de La Candelaria, Tierra del Fuego (Siglos XIX$X X)$. Tesis para optar al grado de Doctora en Arqueología, Universidad Nacional del Centro de la Provincia de Buenos Aires, ciudad.

Guichón, R. 2016. Construyendo preguntas en el camino. Comunidades originarias y científicas. Revista del Museo de Antropología 9 (2):27-36.

Guichón, R.A., P. García Laborde; J.M.B. Motti, M. Martucci, R. Casali, F. Huilinao, M. Maldonado, M. Salamanca, B. Bilte, A. Guevara, C.G. Gallardo Pantoja, M.A. Suarez, M.A. Salerno, L.O. Valenzuela, M.D. D'Angelo del Campo y P.I. Palacio 2015. Experiencias de trabajo conjunto entre investigadores y pueblos originarios. el caso de Patagonia austral. Revista Argentina de Antropología Biológica 17 (2).

Irigaray, L. 1998. Ser Dos. Buenos Aires, Paidós.

Joyce, R.A. 2005. Archaeology of the body. Annual Review of Anthropology 34: 39-158.

Larsen, C.S. 2002. Bioarchaeology: The lives and lifestyles of past people. Journal of Archaeological Research 10 (2):119-166.

Ley Nro. 10316. Creación en ámbito Agencia Córdoba Cultura S.E. del "Registro de Comunidades de Pueblos Indígenas de la Provincia." http://web2.cba.gov. ar/web/leyes.nsf/85a69a561f9ea43d03257234006a8594/ 
d7d2815bbc349bf203257f0f0074493a?OpenDocument (27 de diciembre de 2018).

Martin, D., H.R. Ventura y R. Pérez 2013. Bioarchaeology. An integrated approach to working with human remains. En Manuals in Archaeological Method, Theory and Technique, editado por C. Orser y M. Schiffer, Springer Science, New York.

Menezes Ferreyra, L., M. Montenegro, M.C. Rivolta y J. Nastri 2014. Arqueología, multivocalidad y activación patrimonial en Sudamérica "no somos ventrílocuos". En Multivocalidad y Activaciones Patrimoniales en Arqueología: Perspectivas desde Sudamérica, editado por M.C. Rivolta, M. Montenegro, L. Menezes Ferreira y J. Nastri, pp. 15-29. Fundación de Historia Natural Félix de Azara, Buenos Aires.

Merriman, N. 2004. Involving the public in museum archaeology. En Public Archaeology, editado por N. Merriman, pp. 85-108, Routledge, Londres y Nueva York.

Montenegro, M. 2012. Arqueología en la escuela: experiencias en el sector septentrional del Noroeste argentino. Chungara Revista de Antropologia Chilena 44 (3):487-498.

Nahuelquier, S., C. Huilinao, F. Huilinao, R. A. Guichon, M.S. Caracotche y P. Garcia Laborde 2015. Trabajamos juntos. Antes y después de la ordenanza Municipal de Puerto Santa Cruz 169/09. En La Arqueología Pública en Argentina: Historias, Tendencias y Desafíos en la Construcción de un Campo Disciplinar, editado por M. Fabra, M.M. Montenegro y M. Zabala, pp. 77-92. Editorial de la Universidad Nacional de Jujuy, Jujuy.

Pastor Homs, M.I. 2004. Pedagogía Museística. Nuevas Perspectivas y Tendencias Actuales. Ariel, Barcelona.

Prats, L. 1997. Antropología y Patrimonio. Ariel, Barcelona.

Quezada, L., G. Grundaman, M. Expósito Verdejo y L. Valez 2001. Preparación y Ejecución de Talleres de Capacitación: una Guía Práctica. Serie Proyecto Comunicación y Didáctica. Santo Domingo, http://bibliotecavirtual.clacso.org.ar/Republica_Dominicana/ ccp/20120731051903/prepara.pdf (27 de diciembre de 2018).

Reca, M., M. Sardi, A. Canzani y C. Dominguez 2014. El público opina: estudio acerca de la exhibición de restos humanos en el Museo de La Plata. Revista del Museo de Antropología 7 (1):167-176.
Scattolin, M.C., F. Bugliani, L. Pereyra Domingorena y L.L. Cortés 2005. La señora de los anillos, entre otras tumbas presantamarianas de Yocavil. Intersecciones en Antropología 6:29-41.

Salega, S. y M. Fabra 2017. Variaciones del terreno y cambios entesiales en poblaciones prehispánicas de Córdoba (Argentina). Revista del Museo de Antropología 10 (2):131-136.

Salerno, V. 2013. Arqueología Pública: reflexiones sobre la construcción de un objeto de estudio. Revista Chilena de Antropología 27:7-37.

Seldes, V. 2012. Hacia una Bioarqueología Social. La Quebrada de Humahuaca en Perspectiva Histórica. Editorial Académica Española, Madrid.

Zabala, M. y M. Fabra 2012. Estrechando vínculos entre "comunidades" en torno al patrimonio arqueológico. Las prácticas extensionistas desde un programa de Arqueología Publica. Revista de Arqueología Pública 6:39-53.

Zabala, M., M. Fabra, G.L. Aichino, G.L. y C. De Carli 2016. Reflexiones en torno a los aportes que realiza la Arqueología Pública en la construcción de memorias e identidades locales en el NE de la provincia de Córdoba (Argentina). Revista E + E, Estudios de Extensión en Humanidades 4 (4):8-22.

Zabala, M. e I. Roura Galtes 2007. Veo, Veo ¿qué ves? Los Objetos Patrimoniales como Medios de Comunicación. Serie Cuadernos, Museo de Antropología, Facultad de Filosofía y Humanidades, Universidad Nacional de Córdoba, ciudad.

Zabala, M., I. Roura Galtes y M. Fabra 2006. Educar en Patrimonio: Educar en Valores. Propuesta Didáctica para Interpretar el Patrimonio en el Aula. Serie Cuadernos, Museo de Antropología, Facultad de Filosofía y Humanidades, Universidad Nacional de Córdoba, Córdova.

Zarate, P., C.V. González, S. Salega, y M. Fabra 2017. Osteobiografía de un individuo del sitio Potrerillo de Larreta (Dto. Santa María, Córdoba): aportes desde la Bioarqueología Social. Libro de Resúmenes XIII Jornadas Nacionales de Antropología Biológica, compilado por R.A. Guichón, J.M. B. Motti, P. García Laborde, M. Martucci y L.O. Valenzuela, p.181. Asociación Argentina de Antropología Biológica, Necochea.
${ }^{1}$ Los códigos de ética profesional de diversas instituciones, en particular el ICOM (2004), han considerado de esta manera a los restos humanos y objetos sagrados que los acompañan, que requieren una valoración especial. Argentina adhirió mediante Resolución 1011/05 de la Secretaría de Cultura de la Presidencia de la Nación (Endere y Ayala 2012).

${ }^{2}$ Reconstrucción de comunicación vía redes sociales, aplicación whatsapp.

${ }^{3}$ Para mayor información, consultar https://asociacioncivilapha. wordpress.com/la-red-de-museos-de-apha/

${ }^{4}$ Proyecto "Osteobiografias": las historias que cuentan los cuerpos a partir del registro bioarqueológico (Laguna Mar Chiquita, Córdoba, Argentina). -Convocatoria PROTRI 2015- Directoras: Mariana Fabra y Mariela Zabala.

${ }^{5}$ Los talleres se realizaron en el Museo Aníbal Montes de la localidad de Miramar, en diciembre de 2016, y en el Museo Histórico de La Puerta, en marzo de 2017.

${ }^{6}$ Las escenas fueron realizadas por el Lic. Santiago Druetta, artista plástico y personal de apoyo de CONICET a partir de fotografías ofrecidas por Hugo Giraudo.
${ }^{7}$ Utilizamos imágenes que fueron cedidas para el proyecto por el guía del Museo de Miramar y fotógrafo Hugo Giraudo.

${ }^{8}$ Los dibujos fueron realizados por la artista plástica y gestora cultural Paola Franco.

${ }^{9}$ La Declaratoria de Río Cuarto generó un impacto a largo plazo en la comunidad tanto científica como de pueblos indígenas (ver Canhue 2005, en Endere y Ayala 2012), ya que además de recomendar la no exhibición de restos humanos que se encuentren en museos, mencionaba la importancia de sensibilizar al público sobre este tema, reconocer la sacralidad de los restos humanos y objetos asociados y sitios arqueológicos, así como contar con permisos previos de las comunidades, y adecuar las metodologías arqueológicas a este respeto. También abogaba por la colaboración entre distintas comunidades para lograr las restituciones. El diálogo de saberes que proponemos desde el PAP se fundamenta en parte en estas recomendaciones.

${ }^{10}$ Durante la escritura de este artículo se presentó en Córdoba el 18 de septiembre de 2017 la Ley que crea el Consejo 
de Comunidades de Pueblos Indígenas de la Provincia de Córdoba.

${ }^{11}$ Este taller contó con aval de la Secretaria de Extensión de la Facultad de Filosofía y Humanidades, UNC mediante Resolución del HCD 223/17.

${ }^{12}$ Llegamos a vincularnos con ella a través del director del Museo de Miramar Juan José Scienza que conocía a la mamá de Daniela que era oriunda de Miramar.

${ }^{13} \mathrm{http} / / /$ bit.ly/arqueologia-publica, https://www.youtube.com/ watch?v=quAguMjjDHs\&feature=youtu.be
${ }^{14}$ Se trata de un "repositorio de materiales educativos para la formación y desempeño docente, coordinado por la Universidad Nacional de Córdoba y la Dirección General de Educación Superior de la Provincia de Córdoba”.

${ }^{15}$ Museo de Ciencias Naturales de la Region de Ansenuza "Anibal Montes, Museo Histórico Municipal "San José" Estación Balnearia, Museo Histórico Municipal de La Puerta, Museo Histórico Municipal "La Para", Museo Histórico Municipal de Villa del Rosario y Museo Regional de la colonización piemontesa de Colonia Marina. 